\title{
Management of Tuberculous Infection of the Spine
}

\author{
Pankaj Kandwal ${ }^{1}$, Vijayaraghavan G. ${ }^{2}$, Arvind Jayaswal ${ }^{2}$ \\ ${ }^{1}$ Department of Orthopaedics, All India Institute of Medical Sciences (AIIMS), Rishikesh, India \\ ${ }^{2}$ Department of Orthopaedics, Primus Superspeciality Hospital, New Delhi, India
}

Spinal tuberculosis accounts for nearly half of all cases of musculoskeletal tuberculosis. It is primarily a medical disease and treatment consists of a multidrug regimen for 9-12 months. Surgery is reserved for select cases of progressive deformity or where neurological deficit is not improved by anti-tubercular treatment. Technology refinements and improved surgical expertise have improved the operative treatment of spinal tuberculosis. The infected spine can be approached anteriorly or posteriorly, in a minimally invasive way. We review the various surgical techniques used in the management of spinal tuberculosis with focus on their indications and contraindications.

Keywords: Spinal tuberculosis; Posterior only vertebral column resection; Anterior instrumentation; Posterior instrumentation; Minimal invasive spine surgery

\section{Introduction}

Spinal tuberculosis (TB) continues to be a scourge in the developing world, contributing to spinal deformity and neurological deficit. Tuberculosis of the spine is primarily a medical disease and anti-tubercular treatment (ATT) is the mainstay approach. Surgery is reserved for patients with either mechanical instability or in the presence of neurological deficit.

\section{Short vs. Long Course ATT}

Osteoarticular TB is paucibacillary in nature. Drug therapy involves an extended course of ATT. The duration of ATT in spinal TB is controversial, with a lack of consensus over the optimum therapy duration. Conventional medical treatment of spinal TB ranges from 18-24 months [1]. Although the World Health Organization recommends that pulmonary and extra-pulmonary TB should be treat- ed using a regimen based on a full 6 months of rifampicin (2Isoniazid, Rifampicin, Pyrazinamide, Ethambutol+ 42Isoniazid, Rifampicin) [2], other experts recommend treatment for 9-12 months because of difficulty in assessing the treatment response $[2,3]$.

The prolonged duration of chemotherapy in TB has raised the important issue of drug compliance. Erratic intake of ATT is one of the most common causes for treatment failure and the emergence of acquired drug resistance in TB. The directly observed treatment short (DOTS) course regimen was introduced to enhance the compliance to ATT in cases of pulmonary TB initially, but was extended to osteoarticular TB. Magnetic resonance imaging has detected healed vertebral lesion at the end of 8 months of extended category 1 DOTS regimen in only $35.2 \%$ patients [4].

\section{Surgical Intervention}

Patients with neurological deficit, potential mechanical

\footnotetext{
Received Oct 31, 2015; Accepted Nov 1, 2015

Corresponding author: Pankaj Kandwal

Department of Orthopaedics, All India Institute of Medical Sciences (AIIMS),

Veerbhadra Marg, Rishikesh (UK), 249203, India

Tel: +91-84-7500-0282, Fax: +91-13-5245-2940, E-mail: pankajkandwal27@gmail.com
} 
instability, and kyphosis leading to sagittal imbalance need operative intervention. Indications for surgery are limited to tissue diagnosis in cases of doubtful diagnosis, drainage of an abscess causing difficulty in deglutition and breathing in cervical spine disease, or evacuation of a large paravertebral abscess showing marked increase in size despite 3 to 6 months of multidrug therapy, neural complications that have not responded to drug therapy, deteriorating neurology despite anti-TB therapy, or recurrence of neural complications $[5,6]$.

Surgery is also indicated when the patient presents with a severe kyphosis $\geq 60^{\circ}$ or if the kyphosis is likely to heal with this amount of deformity. Children younger than seven years of age, with three or more affected vertebral bodies in the dorsal or dorsolumbar spine and two or more 'at-risk signs', are likely to have progression of the kyphosis with growth and should undergo correction and fusion $[7,8]$.

The surgical treatment of spine TB has evolved over the past few decades from non-instrumented decompression and fusion to new generation pedicle screws and anterior reconstruction instruments/implants. Use of implants was considered unsafe until Oga et al. [9] reported that, unlike Staphylococcus aureus, Mycobacterium spp. do not form extensive biofilms. Hence, the use of an implant in the setting of a spine TB infection is not a hazard when combined with intensive anti-TB chemotherapy.

\section{Anterior Instrumentation}

Instrumentation in the treatment of spinal TB is essential when the spinal column has been rendered unstable in the process of surgical debridement/decompression, when correction of deformity is anticipated or when the aim is to prevent progression of kyphosis.

Spinal TB is predominantly an anterior disease. Cord compression by granulation tissue, casseous material and abscess is largely from the front. Thus anterior decompression is justified especially in presence of healthy posterior elements. The dorsal spine can be approached anteriorly either by thoracotomy (transthoracic, transpleural) (Fig. 1), or by the extrapleural (anterolateral) approach. Anterior debridement and fusion was popularized by Hodgson et al. [10]. Benli et al. [11] in a series of 100 patients with
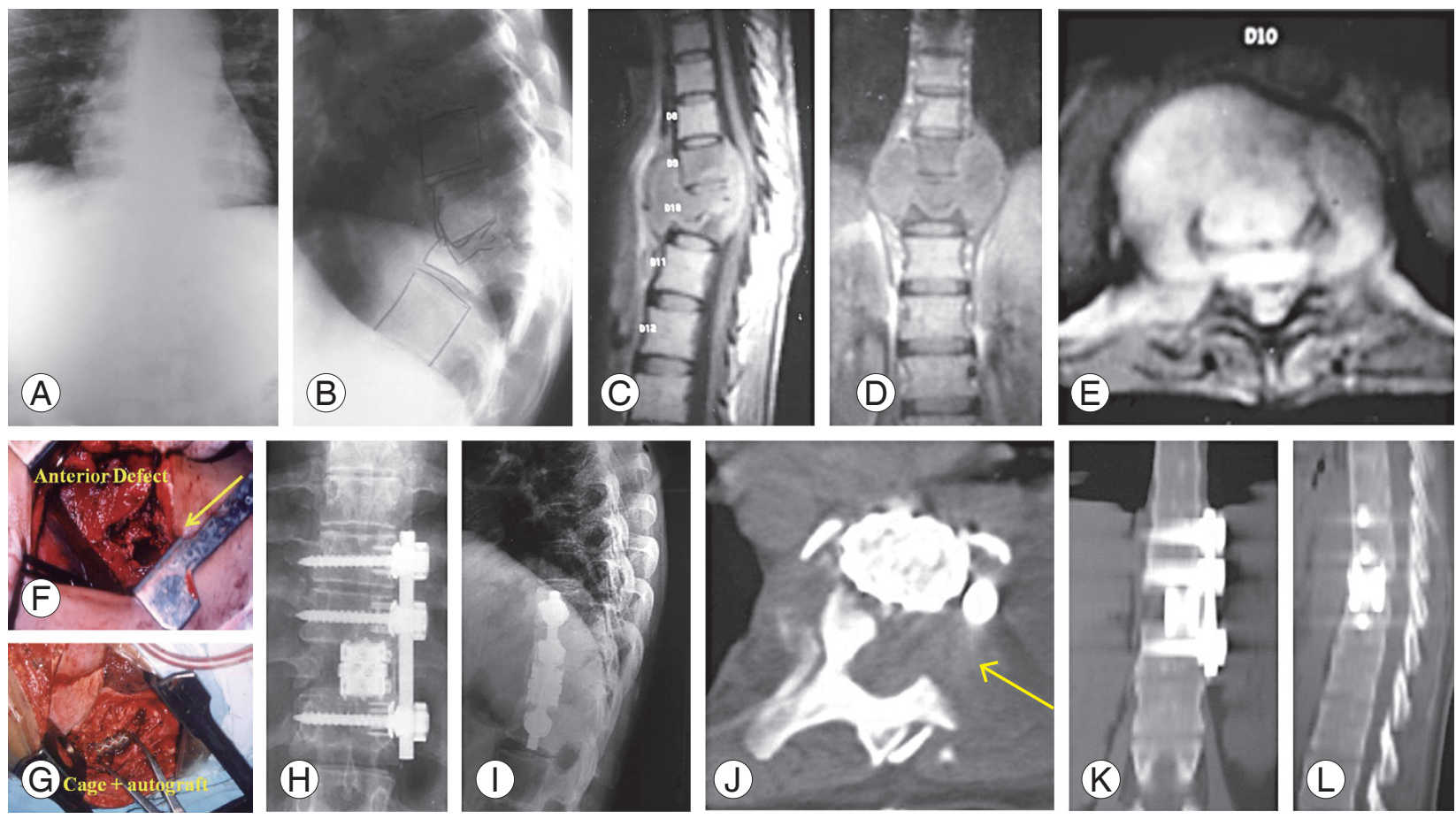

Fig. 1. Preoperative X-rays from a 26 year female with tuberculosis spine D8-10 with paraplegia. (A) Anterior-posterior. (B) Lateral. Preoperative magnetic resonance imaging. (C) T1-weighted saggital. (D) T1-weighted coronal. (E) T2-weighted axial. Intraoperative pictures (F, G). Postoperative X-rays. (H) Antero-posterior. (I) Lateral, following anterior decompression and anterior Instrumentation. Computed tomography scan during follow-up showing good decompression (yellow arrow) (J) and fusion (K, L). 
anterior instrumentation, reported $19.8^{\circ} \pm 7.3^{\circ}$ correction; the preoperative local kyphosis angle was $22.5^{\circ} \pm 11.0^{\circ}$ (range, $10^{\circ}-80^{\circ}$ ) and the postoperative angle was $2.7^{\circ} \pm 3.9^{\circ}$ (range, $0^{\circ}-22^{\circ}$ ). The latter study reported no apparent pseudarthroses and implant failures, and all patients demonstrated clinical healing of $\mathrm{TB}$ without recurrence and reactivation [11]. Use of a mean of $18^{\circ}$ of kyphosis correction was achieved in adult patients after anterior instrumentation surgery during the follow-up period [2]. In another study, kyphotic deformity was corrected by an average of about $16^{\circ}$ using anterior instrumentation and fusion, and in the follow-up period, correction loss was $1^{\circ}$ (6.3\%) [12].

Anterior instrumentation has advantages when compared to posterior instrumentation, as both instrumentation and grafting are done as single-stage surgery through the same incision, which minimizes total blood loss and surgery time without much risk of the graft slipping out due to turning of the patient for posterior instrumentation. The approach also prevents fusing an unnecessarily large number of levels saving segments. However, anterior plate or rods and screws can be used in short-segment disease. As healthy vertebral bodies are necessary above and below the diseased segment to acquire purchase, this system can only be used in mild to moderate kyphosis. Anterior instrumentation can only be used when disease affects the anterior and middle columns only and the posterior column is healthy. In panvertebral disease, anterior instrumentation alone does not provide mechanical stability [13]. In a patient with paraplegia in whom the intercostals muscles are paralyzed (paretic) with a compromised pulmonary function, thoracotomy will increase the risk of postoperative complications [14].

\section{Anterolateral decompression}

In patients with compromised pulmonary reserve, a lateral extrapleural (anterolateral) approach can be done. It allows an easier and better exposure of the spinal cord in a severely kyphotic spine, which is technically difficult to expose by a transthoracic approach [14]. Anterolateral decompression became popular in the era preceding spinal fixation for tubercular spondylitis as it allowed the preservation and stabilization of posterior elements.

\section{Posterior Stabilization}

Posterior instrumented fusion can be done after anterior decompression either as a two-stage or single-stage procedure [15]. Alternatively, the cord can be decompressed by posterior, posterolateral (Fig. 2) or transpedicular approach along with posterior stabilization/instrumentation [16-19]. The advent of pedicle screw instrumentation and the development of the extended posterior approaches has allowed the surgeon to perform anterior debridement and reconstruction from behind, and has led many surgeons to opt for posterior-only surgery. It further avoids the possible hazards of violating the thoracic or abdominal
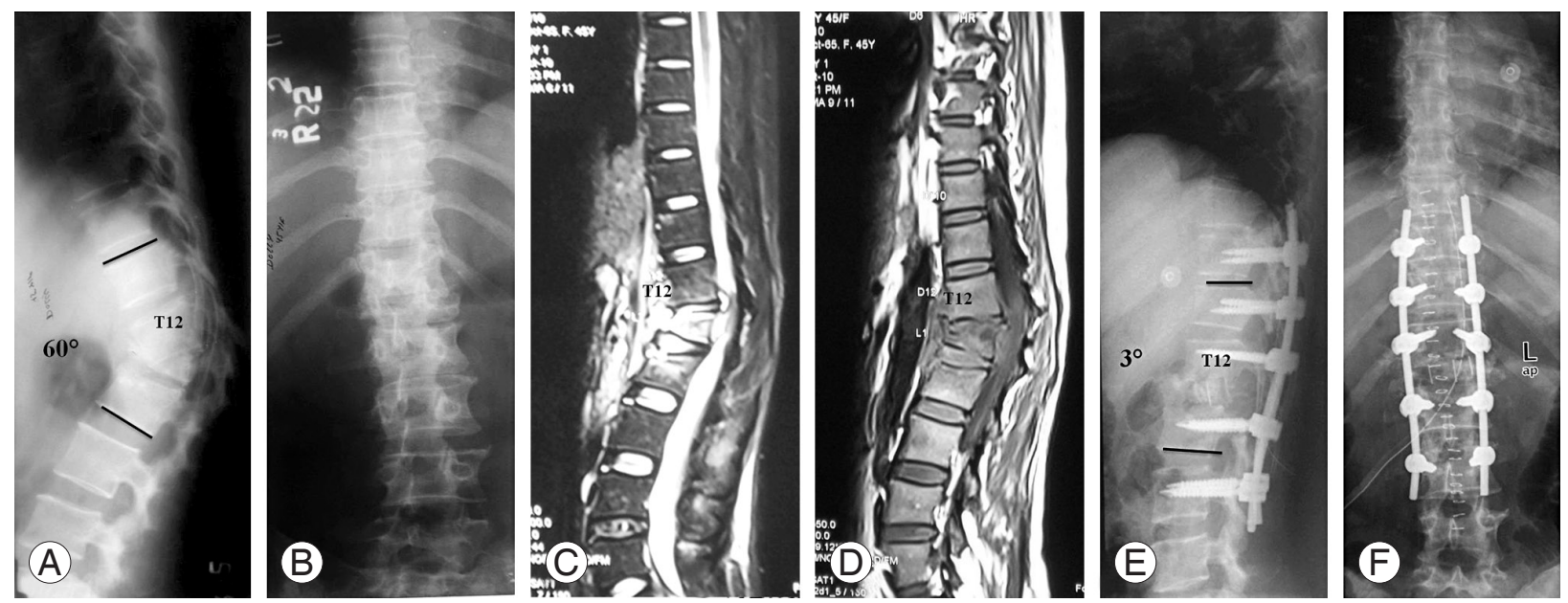

Fig. 2. A case of a 40 year female with active tubercular spondylitis T12-L2 with thoracolumbar kyphosis. Preoperative X-rays. (A) Lateral view (B) Anterior-posterior view. Preoperative magnetic resonance imaging. (C) Short tau inversion recovery. (D) T1-weighted images showing active disease. Postoperative X-rays (E) Lateral view, (F) Anterior-posterior view following pedicle screw instrumentation, posterolateral decompression and reconstruction with a polyether ether ketone cage. 
cavities [20]. Spinal TB is associated with osteopenia or soft vertebral bodies. Posterior instrumentation in these cases is justified by the fact that posterior elements are relatively healthier when compared to the vertebral body [5]. The recent literature is replete with use of posterior instrumentation in spinal TB [16-18,21-25]. Whether the anterior approach is superior to the posterior approach is still debatable among surgeons. A comparative study of surgical management by the posterior only and combined posterior and anterior approaches for thoracic spinal TB in the elderly concluded that the posterior only procedure obtained better clinical outcomes than combined posterior and anterior surgeries [25]. A previous study compared the anterior and posterior approach in the treatment of thoracic and thoracolumbar TB; the approaches were comparable with regards to duration of surgery, blood loss, fusion and functional outcomes but the posterior approach was associated with significantly better kyphus correction when compared to the anterior approach [26]. Another comparative study concluded that the posterior approach may be superior to anterior instrumentation to correct a deformity and maintain the correction [27].

Controversy among surgeons exists if anterior approach is superior to posterior or vice versa. Zhang et al. [25] in a comparative study of surgical management by posterior only and combined posterior and anterior approaches for thoracic TB in the elderly concluded that posterior ap- proach only procedure obtained better clinical outcomes than combined posterior and anterior surgeries.

The authors in a previous study compared anterior and posterior approach in the treatment of thoracic and thoracolumbar $\mathrm{TB}$ and found that both the approaches are comparable with regards to duration of surgery, blood loss, fusion and functional outcomes but posterior approach was associated with significantly better kyphus correction when compared to anterior [26]. Similarly Ma et al. [27] in a comparative study concluded that posterior approach may be superior to anterior instrumentation to correct deformity and maintain that correction.

\section{Vertebral column resection in spinal TB}

Continuous destruction of the anterior column and progressive anterior collapse of the spine by TB despite ATT may result in kyphosis. After healing of the spinal infection, the post-TB kyphosis in adults may be static, but in children kyphosis progresses dramatically in almost $40 \%$ of cases [12]. Angular kyphosis secondary to TB may progress even after healing of the disease and may lead to sagittal plane imbalance, cardiopulmonary compromise and late onset paraplegia. Fixed angular deformity in the sagittal plane resulting from healed TB requires three column osteotomy for adequate restoration of sagittal balance [28].
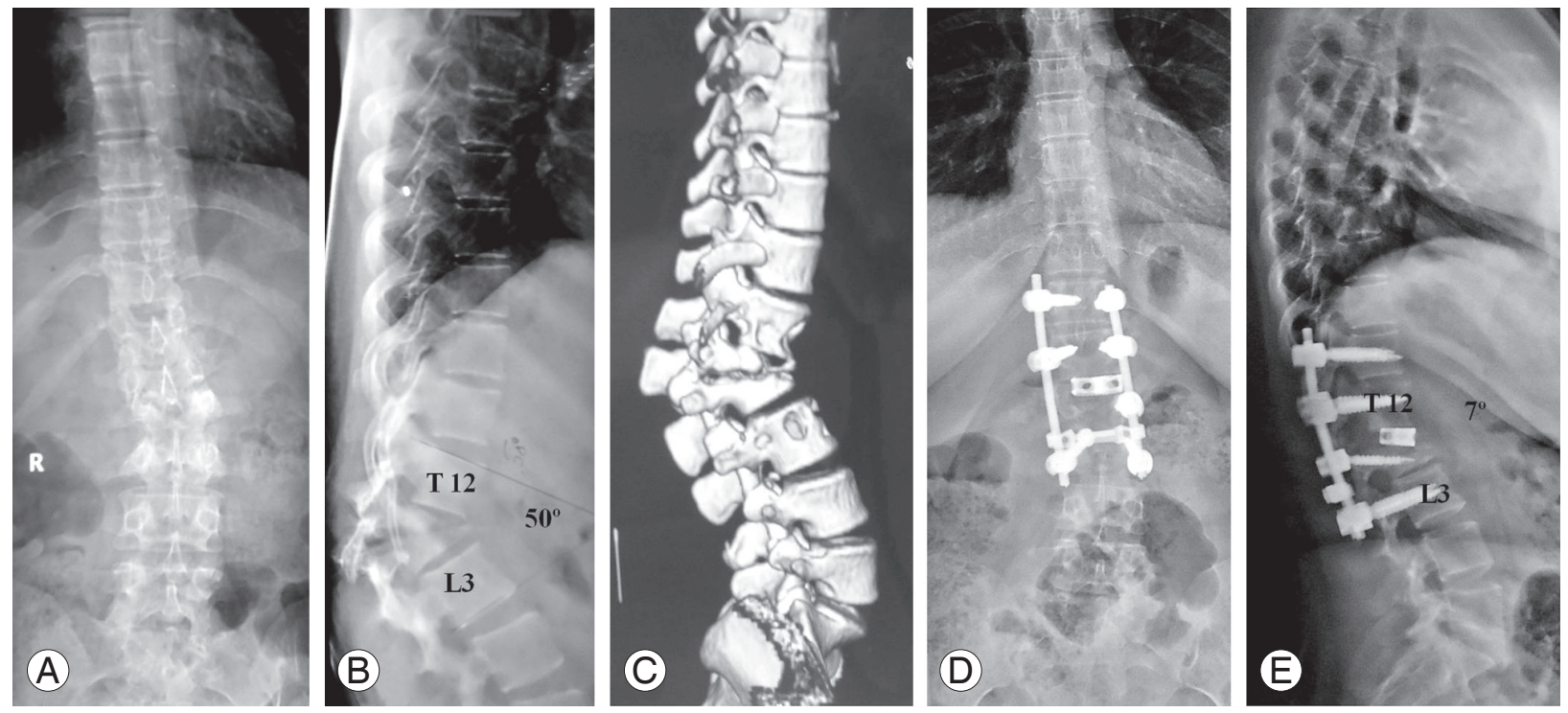

Fig. 3. Healed tuberculosis with fixed thoracolumbar kyphosis in a 25-year-old male. Preoperative X-rays. (A) Anterior-posterior view and (B) Lateral view. Preoperative computed tomography scan with three-dimensional reconstruction. (C) Postoperative X-rays. (D) Anterior-posterior view and (E) Lat view following posterior only vertebral column resection. 

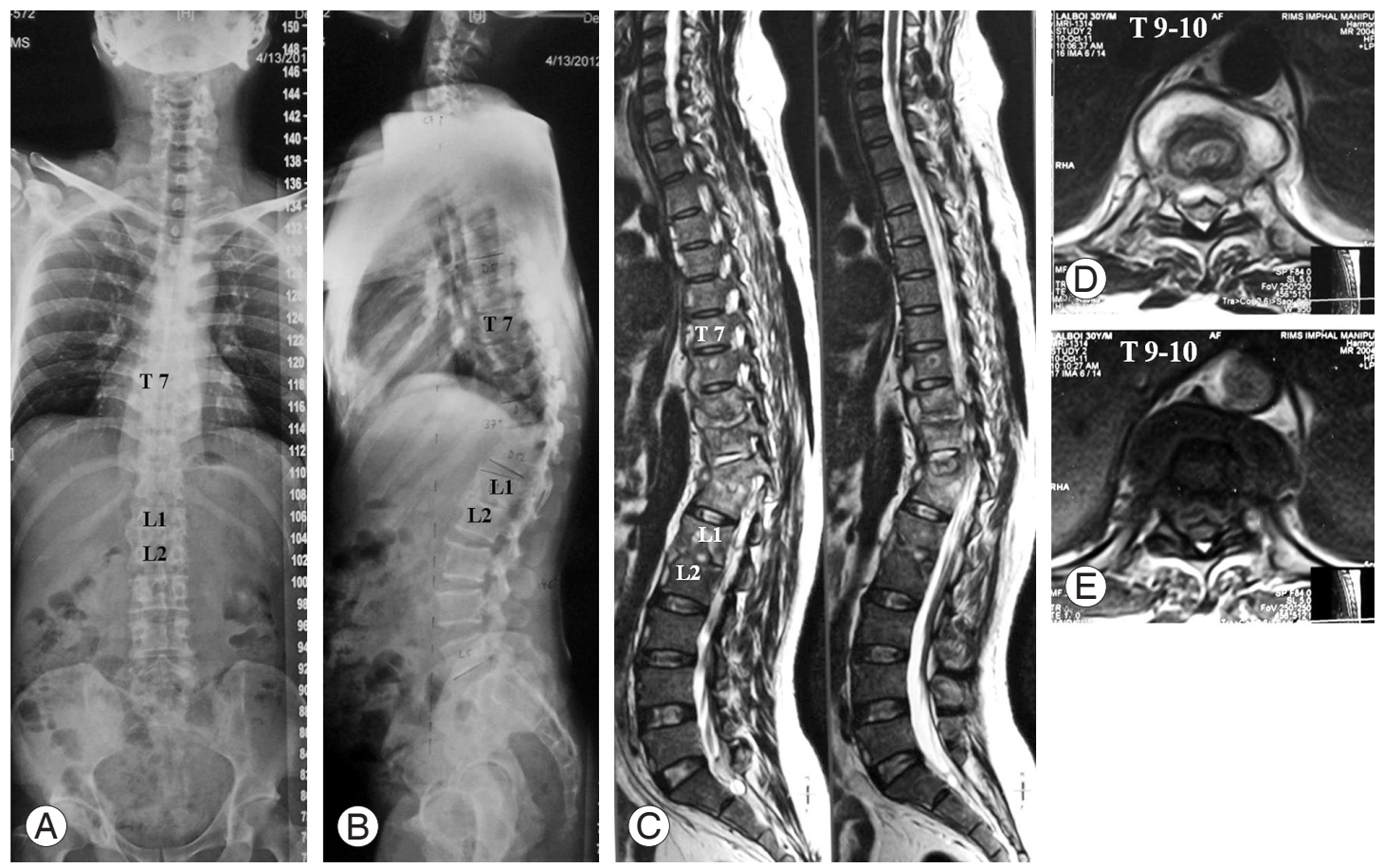

Fig. 4. Multilevel spinal tuberculosis in a 30-year-old male. Preoperative X-rays. (A) Anterio-posterior view and (B) Lateral view. Preoperative magnetic resonance imaging. (C) T2-weighted sagittal. (D) T2-weighted axial and (E) T1-weighted Axial sections showing cord compression at the T9-10 level.
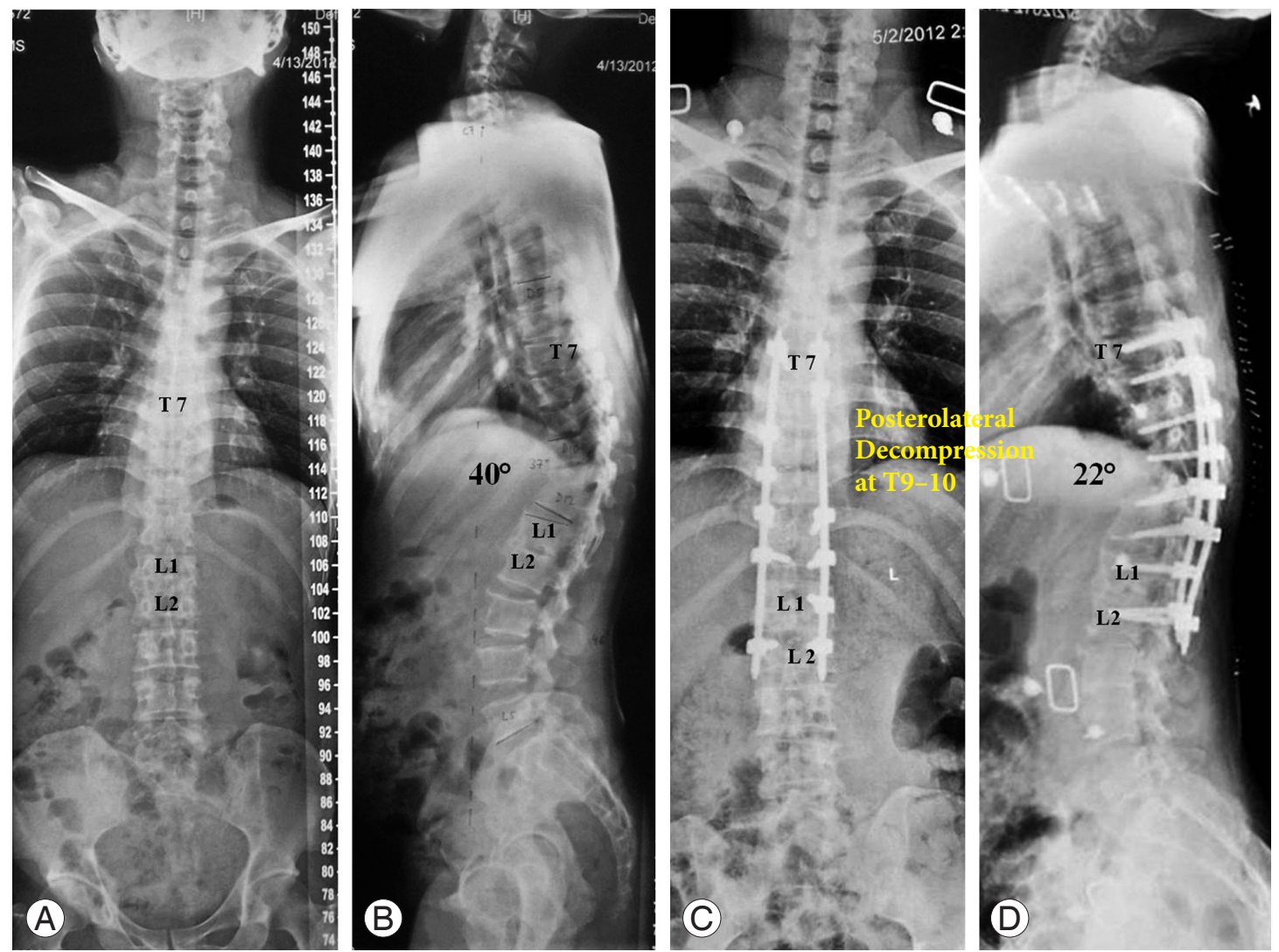

Fig. 5. Multilevel spinal tuberculosis in a 30-year-old male. Preoperative X-rays. (A) Anterior-posterior view and (B) Lateral view. Postoperative X-rays. (C) Anterior-posterior view and (D) lateral view following percutanous pedicle screws and posterolateral decompression at T9-10 level using retractor system. 
Since the advent of posterior only vertebral column resection (PVCR) by Suk et al. [29], various authors have attempted PVCR or similar osteotomies for tubercular deformities of spine. Good results have been reported [20,29-35]. A study involving a series of 17 patients reported an average kyphosis correction rate of $68.7 \% \pm 6.5 \%$ with an average correction loss as $3.3^{\circ}$ [30]. Similarly, $\mathrm{Lu}$ et al. [33] reported $80.1 \%$ correction of kyphosis. Vertebral column resection is an osteotomy that destabilizes the vertebral column. It is a technically challenging procedure. The complication rate can be as high as $40 \%$ [36] which includes dural tear, neurological deficit (temporary and permanent) and lung-related complications apart from prolonged operation time and blood loss. The authors recommend this surgical procedure only for severe kyphosis either in active or healed disease (Fig. 3).

\section{Minimal invasive spine surgery in spinal TB}

Ever since the introduction of a minimally invasive spine surgery [37], the approach has become one of the standard treatment procedures for various degenerative spinal disorders.

\section{Thoracoscopic decompression and anterior instru- mentation}

Video-assisted thoracoscopic surgery (VATS) was developed to decrease the approach related morbidity associated with thoracotomy. VATS is a valuable alternative to conventional thoracotomy with minimal morbidity. VATS has documented comparable results to open procedures in scoliosis, but few studies have addressed its role in spinal TB. Few authors have explored the option of VATS in anterior decompression and instrumentation [38-40]. The senior author (A.J.) has been selectively doing diverse thoracoscopic procedures that include abscess drainage, grafting and anterior decompression with or without instrumentation. Good clinical, neurological outcome and fusion have been reported following VATS in tubercular spondylitis [39]. Long term follow-ups concluded that VATS-assisted surgical decompression can be a safe and effective technique for anterior debridement and fusion in tuberculosis of the dorsal spine to achieve neurological recovery with reduced morbidity, blood loss and hospital stay compared to thoracotomy $[41,42]$.
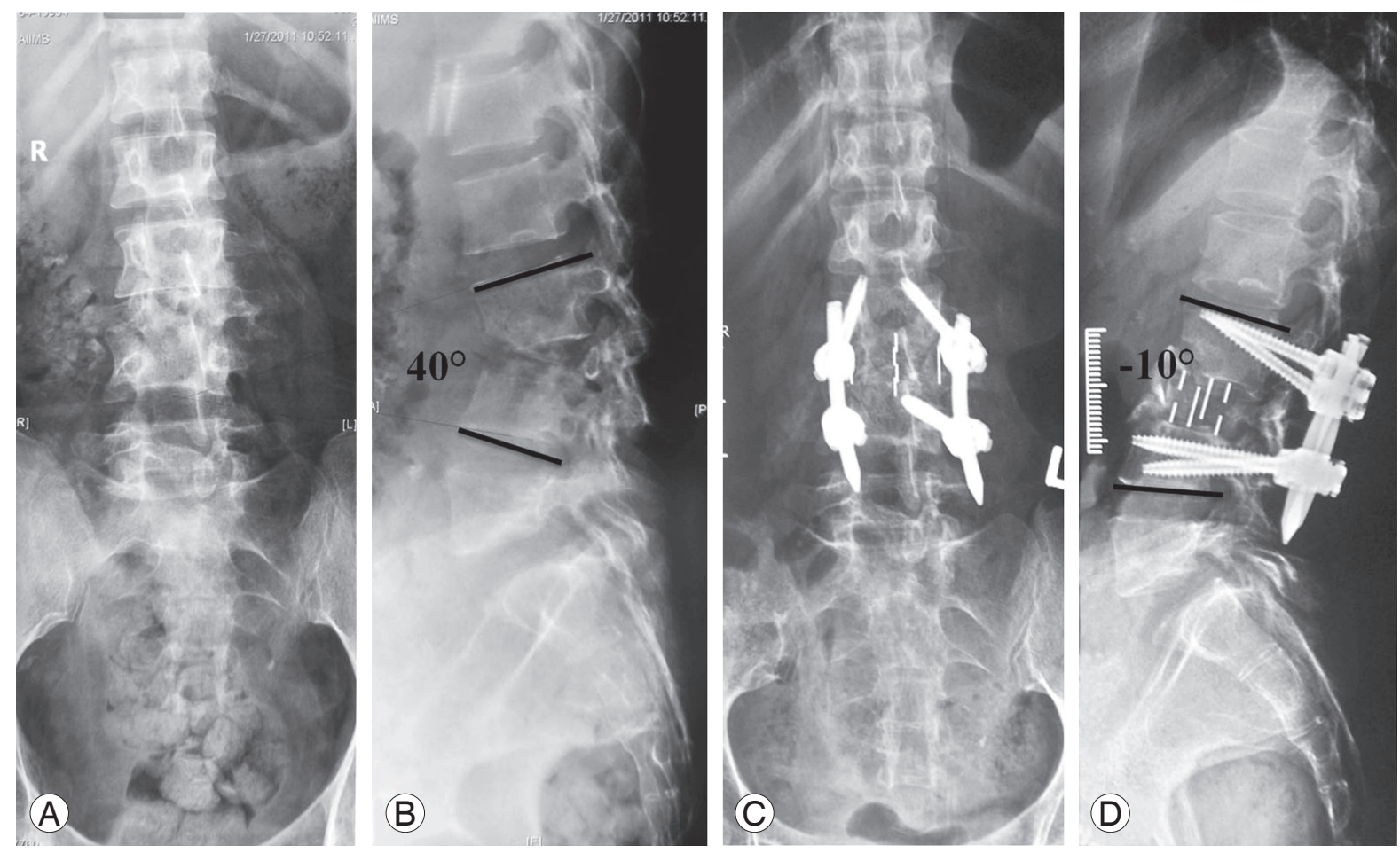

Fig. 6. Tubercular spondylitis $L 2-L 4$, with complete destruction of $L 3$ vertebra in a 30-year-old female. Preoperative X-rays. (A) Anteriorposterior view and (B) lateral view. Percutanous pedicle screws fixation in prone position followed by decompression, interbody fusion using DLIF (Medtronics Inc.). Postoperative X rays. (C) Anterior-posterior view and (D) lateral view. 

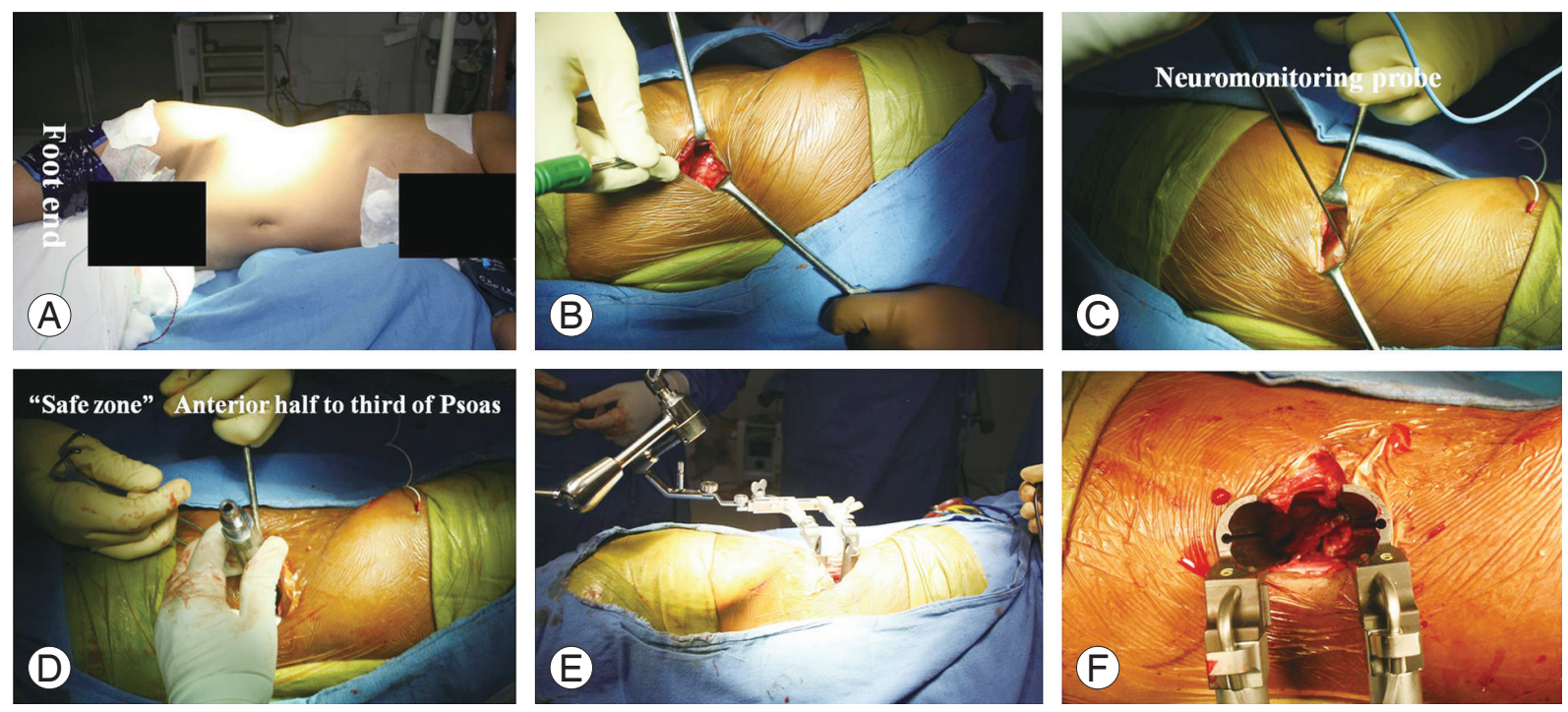

Fig. 7. Tubercular spondylitis $L 2-L 4$, with complete destruction of $L 3$ vertebra in a 30-year-old female. Intra operative photographs of DLIF. Lateral position (A) with bolster below the flank region. Five centimeter incision on the lateral aspect (B). Insertion of neuromonitoring probe (C). Serial dilators were inserted (D) followed by a retractor device (E, F).

\section{Percutanous pedicle screw fixation and posterolateral decompression using tubular/mini-open retractor system}

Posterolateral endoscopic debridement and irrigation for tubercular spondylodiscitis was performed initially [43]. The versatility of percutanous pedicle screw instrumentation allows it to stabilize both the thoracic and lumbar spine. Decompression of spinal cord/thecal sac can be done simultaneously by facetectomy using retractor system and interbody fusion can be done either by the transforaminal approach (Figs. 4, 5) or direct lateral approach (Figs. 6, 7). Few authors have tried and documented good fusion rates with encouraging functional outcome following percutanous pedicle screw $[44,45]$. The authors recommend percutanous instrumentation in multisegmental spinal TB with instability.

\section{Conclusions}

Spinal TB is a debatable condition regarding optimal ATT duration and the approach used for surgical decompression. With the introduction of newer instrumentation, anterior decompression by conventional thoracotomy has given way to VATS assisted anterior decompression and posterolateral decompression along with pedicle screw instrumentation. On the other hand, percutanous pedicle screws with minimal access decompression can be a valuable alternative to traditional open procedures in selected cases.

\section{Conflict of Interest}

No potential conflict of interest relevant to this article was reported.

\section{References}

1. Rajasekaran S, Khandelwal G. Drug therapy in spinal tuberculosis. Eur Spine J 2013;22 Suppl 4:587-93.

2. World Health Organization. Guidelines for treatment of tuberculosis [Internet]. Geneva: World Health Organization; 2016 [cited 2015 Jul 27]. Available from: http://www.who.int/tb/publications/2010/9789241547833/en/.

3. Blumberg HM, Burman WJ, Chaisson RE, et al. American Thoracic Society/Centers for Disease Control and Prevention/Infectious Diseases Society of America: treatment of tuberculosis. Am J Respir Crit Care Med 2003;167:603-62.

4. Jain AK, Srivastava A, Saini NS, Dhammi IK, Sreenivasan R, Kumar S. Efficacy of extended DOTS category I chemotherapy in spinal tuberculosis based on MRI-based healed status. Indian J Orthop 2012; 46:633-9. 
5. Tuli SM. Historical aspects of Pott's disease (spinal tuberculosis) management. Eur Spine J 2013;22 Suppl 4:529-38.

6. Tuli SM. Tuberculosis of the spine: a historical review. Clin Orthop Relat Res 2007;460:29-38.

7. Rajasekaran S. The problem of deformity in spinal tuberculosis. Clin Orthop Relat Res 2002;(398):8592.

8. Jain AK. Tuberculosis of the spine: a fresh look at an old disease. J Bone Joint Surg Br 2010;92:905-13.

9. Oga M, Arizono T, Takasita M, Sugioka Y. Evaluation of the risk of instrumentation as a foreign body in spinal tuberculosis: clinical and biologic study. Spine (Phila Pa 1976) 1993;18:1890-4.

10. Hodgson AR, Stock FE, Fang HS, Ong GB. Anterior spinal fusion. The operative approach and pathological findings in 412 patients with Pott's disease of the spine. Br J Surg 1960;48:172-8.

11. Benli IT, Kaya A, Acaroglu E. Anterior instrumentation in tuberculous spondylitis: is it effective and safe? Clin Orthop Relat Res 2007;460:108-16.

12. Rajasekaran $\mathrm{S}$. The natural history of post-tubercular kyphosis in children. Radiological signs which predict late increase in deformity. J Bone Joint Surg Br 2001;83:954-62.

13. Jain AK, Jain S. Instrumented stabilization in spinal tuberculosis. Int Orthop 2012;36:285-92.

14. Jain AK. Treatment of tuberculosis of the spine with neurologic complications. Clin Orthop Relat Res 2002;(398):75-84.

15. Jain AK, Dhammi IK, Prashad B, Sinha S, Mishra P. Simultaneous anterior decompression and posterior instrumentation of the tuberculous spine using an anterolateral extrapleural approach. J Bone Joint Surg Br 2008;90:1477-81.

16. Pang X, Wu P, Shen X, Li D, Luo C, Wang X. Onestage posterior transforaminal lumbar debridement, 360 degrees interbody fusion, and posterior instrumentation in treating lumbosacral spinal tuberculosis. Arch Orthop Trauma Surg 2013;133:1033-9.

17. Kim DJ, Yun YH, Moon SH, Riew KD. Posterior instrumentation using compressive laminar hooks and anterior interbody arthrodesis for the treatment of tuberculosis of the lower lumbar spine. Spine (Phila Pa 1976) 2004;29:E275-9.

18. Luo C, Wang X, Wu P, Ge L, Zhang H, Hu J. Singlestage transpedicular decompression, debridement, posterior instrumentation, and fusion for thoracic tuberculosis with kyphosis and spinal cord compression in aged individuals. Spine J 2016;16:154-62.

19. Pang X, Shen X, Wu P, Luo C, Xu Z, Wang X. Thoracolumbar spinal tuberculosis with psoas abscesses treated by one-stage posterior transforaminal lumbar debridement, interbody fusion, posterior instrumentation, and postural drainage. Arch Orthop Trauma Surg 2013;133:765-72.

20. Rajasekaran S. Kyphotic deformity in spinal tuberculosis and its management. Int Orthop 2012;36:35965.

21. Zeng H, Zhang P, Shen X, et al. One-stage posterioronly approach in surgical treatment of single-segment thoracic spinal tuberculosis with neurological deficits in adults: a retrospective study of 34 cases. BMC Musculoskelet Disord 2015;16:186.

22. Sundararaj GD, Behera S, Ravi V, Venkatesh K, Cherian VM, Lee V. Role of posterior stabilisation in the management of tuberculosis of the dorsal and lumbar spine. J Bone Joint Surg Br 2003;85:100-6.

23. Laheri VJ, Badhe NP, Dewnany GT. Single stage decompression, anterior interbody fusion and posterior instrumentation for tuberculous kyphosis of the dorso-lumbar spine. Spinal Cord 2001;39:429-36.

24. Lee SH, Sung JK, Park YM. Single-stage transpedicular decompression and posterior instrumentation in treatment of thoracic and thoracolumbar spinal tuberculosis: a retrospective case series. J Spinal Disord Tech 2006;19:595-602.

25. Zhang HQ, Li JS, Zhao SS, et al. Surgical management for thoracic spinal tuberculosis in the elderly: posterior only versus combined posterior and anterior approaches. Arch Orthop Trauma Surg 2012;132: 1717-23.

26. Garg B, Kandwal P, Nagaraja UB, Goswami A, Jayaswal A. Anterior versus posterior procedure for surgical treatment of thoracolumbar tuberculosis: A retrospective analysis. Indian J Orthop 2012;46:165-70.

27. Ma YZ, Cui X, Li HW, Chen X, Cai XJ, Bai YB. Outcomes of anterior and posterior instrumentation under different surgical procedures for treating thoracic and lumbar spinal tuberculosis in adults. Int Orthop 2012;36:299-305.

28. Boachie-Adjei O, Papadopoulos EC, Pellise F, et al. Late treatment of tuberculosis-associated kyphosis: literature review and experience from a SRS-GOP 
site. Eur Spine J 2013;22 Suppl 4:641-6.

29. Suk SI, Kim JH, Kim WJ, Lee SM, Chung ER, Nah $\mathrm{KH}$. Posterior vertebral column resection for severe spinal deformities. Spine (Phila Pa 1976) 2002;27: 2374-82.

30. Zhou T, Li C, Liu B, Tang X, Su Y, Xu Y. Analysis of 17 cases of posterior vertebral column resection in treating thoracolumbar spinal tuberculous angular kyphosis. J Orthop Surg Res 2015;10:64.

31. Deng Y, Lv G, An HS. En bloc spondylectomy for the treatment of spinal tuberculosis with fixed and sharply angulated kyphotic deformity. Spine (Phila Pa 1976) 2009;34:2140-6.

32. Wang Y, Zhang Y, Zhang X, et al. Posterior-only multilevel modified vertebral column resection for extremely severe Pott's kyphotic deformity. Eur Spine J 2009;18:1436-41.

33. Lu G, Wang B, Li Y, Li L, Zhang H, Cheng I. Posterior vertebral column resection and intraoperative manual traction to correct severe post-tubercular rigid spinal deformities incurred during childhood: minimum 2-year follow-up. Eur Spine J 2015;24:58693.

34. Rajasekaran S, Vijay K, Shetty AP. Single-stage closing-opening wedge osteotomy of spine to correct severe post-tubercular kyphotic deformities of the spine: a 3 -year follow-up of 17 patients. Eur Spine J 2010;19:583-92.

35. Zhang HQ, Li JS, Liu SH, et al. The use of posterior vertebral column resection in the management of severe posttuberculous kyphosis: a retrospective study and literature review. Arch Orthop Trauma Surg 2013;133:1211-8.

36. Kim SS, Cho BC, Kim JH, et al. Complications of posterior vertebral resection for spinal deformity.
Asian Spine J 2012;6:257-65.

37. Foley KT, Holly LT, Schwender JD. Minimally invasive lumbar fusion. Spine (Phila Pa 1976) 2003;28: S26-35.

38. Huang TJ, Hsu RW, Chen SH, Liu HP. Video-assisted thoracoscopic surgery in managing tuberculous spondylitis. Clin Orthop Relat Res 2000;(379):14353.

39. Jayaswal A, Upendra B, Ahmed A, Chowdhury B, Kumar A. Video-assisted thoracoscopic anterior surgery for tuberculous spondylitis. Clin Orthop Relat Res 2007;460:100-7.

40. Kapoor SK, Agarwal PN, Jain BK Jr, Kumar R. Videoassisted thoracoscopic decompression of tubercular spondylitis: clinical evaluation. Spine (Phila Pa 1976) 2005;30:E605-10.

41. Kapoor S, Kapoor S, Agrawal M, Aggarwal P, Jain BK Jr. Thoracoscopic decompression in Pott's spine and its long-term follow-up. Int Orthop 2012;36:331-7.

42. Lu G, Wang B, Li J, Liu W, Cheng I. Anterior debridement and reconstruction via thoracoscopy-assisted mini-open approach for the treatment of thoracic spinal tuberculosis: minimum 5-year follow-up. Eur Spine J 2012;21:463-9.

43. Ito M, Sudo H, Abumi K, et al. Minimally invasive surgical treatment for tuberculous spondylodiscitis. Minim Invasive Neurosurg 2009;52:250-3.

44. Kandwal P, Garg B, Upendra B, Chowdhury B, Jayaswal A. Outcome of minimally invasive surgery in the management of tuberculous spondylitis. Indian J Orthop 2012;46:159-64.

45. Garg N, Vohra R. Minimally invasive surgical approaches in the management of tuberculosis of the thoracic and lumbar spine. Clin Orthop Relat Res 2014;472:1855-67. 